\title{
Comparative effectiveness of tocilizumab versus TNF inhibitors as monotherapy or in combination with conventional synthetic disease-modifying antirheumatic drugs in patients with rheumatoid arthritis after the use of at least one biologic disease- modifying antirheumatic drug: analyses from the pan- European TOCERRA register collaboration
}

\author{
Kim Lauper, ${ }^{1,2}$ Dan C Nordström, ${ }^{3}$ Karel Pavelka, ${ }^{4}$ Maria Victoria Hernández, ${ }^{5}$ \\ Tore K Kvien, ${ }^{6}$ Eirik Klami Kristianslund ${ }^{6}$ Maria Jose Santos, ${ }^{7}$ Žiga Rotar, ${ }^{8}$ \\ Florenzo lannone, ${ }^{9}$ Catalin Codreanu, ${ }^{10}$ Galina Lukina, ${ }^{11}$ Sara L Gale, ${ }^{12}$ \\ Khaled Sarsour, ${ }^{12}$ Yves Luder, ${ }_{13}^{13}$ Delphine Sophie Courvoisier, ${ }_{1}^{1}$ Cem Gabay ${ }^{1,2}$
}

\begin{abstract}
Handling editor Josef $S$ Smolen

- Additional material is published online only. To view, please visit the journal online (http://dx.doi.org/10.1136/ annrheumdis-2017-212845).

For numbered affiliations see end of article.
\end{abstract}

Correspondence to Professor Cem Gabay, Division of Rheumatology, Geneva University Hospitals, Geneva 1205, Switzerland; cem.gabay@hcuge.ch

This manuscript is based on work previously presented at EULAR 2017 congress and published as a conference abstract: Lauper K, Nordström D. Pavelka K et al. SAT0206 Retention of tocilizumab as monotherapy versus TNF inhibitors with conventional synthetic DMARDS in rheumatoid arthritis patients with inadequate response to TNF inhibitors: a study from the TOCERRA collaboration. Annals of the Rheumatic Diseases 2017;76:850-851.

Received 14 December 2017 Accepted 24 April 2018

Published Online First 5 May 2018

\section{Check for updates}

To cite: Lauper $\mathrm{K}$ Nordström DC, Pavelka K et al. Ann Rheum Dis 2018;77:1276-1282.

\section{ABSTRACT}

Objective To compare the effectiveness of tocilizumab (TCZ) and tumour necrosis factor (TNF) inhibitors (TNFi) as monotherapy or in combination with conventional synthetic disease-modifying antirheumatic drugs (DMARDs) in patients with rheumatoid arthritis (RA) after the use of at least one biologic DMARD (bDMARD).

Methods We included patients with RA having used at least one bDMARD from 10 European registries. We compared drug retention using Kaplan-Meier and Cox models and Clinical Disease Activity Index (CDAI) change over time with mixed-effects models for longitudinal data. The proportions of CDAl remission and low disease activity (LDA) at 1 year were compared using LUNDEX correction. Results 771 patients on TCZ as monotherapy (TCZ mono), 1773 in combination therapy (TCZ combo), 1404 on TNFi as monotherapy (TNFi mono) and 4660 in combination therapy (TNFi combo) were retrieved. Crude median retention was higher for TCZ mono $(2.31$ years, $95 \% \mathrm{Cl}$ 2.07 to 2.61 ) and TCZ combo (1.98 years, $95 \% \mathrm{Cl} 1.83$ to 2.11$)$ than TNFi combo $(1.37$ years, $95 \% \mathrm{Cl} 1.30$ to 1.45) and TNFi mono (1.31 years, $95 \% \mathrm{Cl} 1.18$ to 1.47 ). In a country and year of treatment initiation-stratified, covariate-adjusted analysis, hazards of discontinuation were significantly lower among patients on TCZ mono or combo compared with patients on TNFi mono or combo, and TNFi combo compared with TNFi mono, but similar between TCZ mono and combo. Average adjusted CDAl change was similar between groups. CDAI remission and LDA rates were comparable between groups.

Conclusion With significantly longer drug retention and similar efficacy to TNFi combo, TCZ mono or combo are reasonable therapeutic options in patients with inadequate response to at least one bDMARD.

\section{INTRODUCTION}

Rheumatoid arthritis (RA) is a chronic systemic autoimmune disease characterised by joint inflammation and structural damage. The management of RA has dramatically changed with the use of biologic disease-modifying antirheumatic drugs (bDMARDs). Tocilizumab (TCZ) is a humanised anti-interleukin (IL)-6 receptor antibody that has shown efficacy in reducing signs and symptoms of RA and in preventing the progression of structural damage and loss of function. ${ }^{1-6}$ TCZ is licensed for the treatment of patients with RA with inadequate response to conventional synthetic DMARDs (csDMARDs) and/or bDMARDs. ${ }^{7}$ Most international recommendations advocate the use of bDMARDs in combination with methotrexate (MTX) or other csDMARDs in case MTX is not tolerated or contraindicated. ${ }^{8}$ However, data derived from various patient registries show that bDMARDs are prescribed as monotherapy in up to $30 \%$ of patients, due to patient's preference or occurrence of intolerance to csDMARDs. ${ }^{9-16}$ The ACT-RAY study examined the efficacy and safety of switching to TCZ monotherapy or adding TCZ to MTX in patients with active disease despite MTX therapy. The results at 24 weeks showed that efficacy was largely similar in both treatment arms, ${ }^{17}$ but this first analysis and later follow-up during 2 years overall suggested that TCZ performed better in combination with MTX than as monotherapy. ${ }^{18} 19$ In a 52-week prospective, randomised controlled study, adding TCZ to MTX more rapidly achieved remission than switching to TCZ monotherapy, in patients with RA refractory to MTX. ${ }^{20}$ In the FUNCTION randomised placebo-controlled trial in early arthritis, the combination of MTX and TCZ seemed to be more efficacious than TCZ in monotherapy, but the study was not powered to detect difference between these groups. ${ }^{21}$ On the other hand, a study combining several European registries found that TCZ as monotherapy (TCZ mono) had similar effectiveness as compared with TCZ in combination with MTX and/or csDMARDs when assessed as changes in Clinical Disease Activity Index (CDAI) and Disease Activity Score 
28 (DAS28) from baseline values. ${ }^{9}$ The ADACTA study demonstrated in a head-to-head randomised controlled trial setting that TCZ monotherapy was superior to adalimumab monotherapy for reduction of signs and symptoms of RA in patients for whom MTX was deemed inappropriate. ${ }^{22}$ However, one of the criticisms of this study is that adalimumab was used as monotherapy, which does not represent the best comparator since TNF inhibitors (TNFi) are notoriously more efficacious when used in combination with MTX. ${ }^{23-25}$ Since TCZ is largely used as a second-line bDMARD in numerous countries, we decided to compare the effectiveness of TCZ and TNFi as monotherapy or in combination with csDMARDs in patients with inadequate responses to at least one bDMARD followed longitudinally in 10 European registries, with a special interest in the comparison between TCZ mono and TNFi in combination with csDMARDs (TNFi combo).

\section{METHODS}

The TOCERRA collaboration of registries (TOcilizumab Collaboration of European Registries in RA) is an investigator-led, industry-supported project aiming at evaluating clinical aspects of TCZ use in patients with RA. Each registry obtained ethical approval for the use of anonymised data for research in their local ethics committee. TOCERRA includes data from 10 countries (see online supplementary table S1). All patients included in the different registries and starting treatment with TCZ or any TNFi between 16 January 2009 and 1 January 2017 were considered eligible for the present study. Inclusion criteria were diagnosis of RA established by a rheumatologist, being aged 18 years or more, having used at least one bDMARD, baseline information on prior use of bDMARDs or csDMARDs and information on concomitant use of csDMARDs. When patients had several treatment courses with either TCZ or TNFi, all treatment courses were used and statistical models included a stochastic term to account for the non-independence of the data.

\section{Exposure of interest}

bDMARDs were classified either as monotherapy or in combination therapy with any csDMARDs, depending on the presence of concomitant csDMARDs at baseline. The main exposures of interest were TCZ or TNFi as monotherapy or in combination with one or several csDMARDs. We also performed three secondary analyses. In the first, we carried out additional detailed analysis between TCZ mono and TNFi combo. In another secondary analysis, patients treated with TNFi and MTX as the only csDMARD were further categorised as having low-dose MTX (<10 mg/week), medium dose (10-15 mg/ week) versus high dose ( $>15 \mathrm{mg} /$ week), yielding four groups (TCZ mono, TNFi combo MTX low dose, TNFi combo MTX medium dose, TNFi combo MTX high dose). Finally, patients treated with TNFi were categorised by their type of concomitant csDMARD (only MTX, MTX + another csDMARD, other csDMARD without MTX).

\section{Study outcomes}

Our main focus was drug retention and the change of disease activity in terms of CDAI following initiation of bDMARDs.

Drug retention reflects both effectiveness and tolerance of a drug and is reliably assessed in all registries. ${ }^{2627}$ It was defined as the time from the start date of TCZ or TNFi treatment until the treatment discontinuation date plus one dispensation interval. If treatment had not been discontinued, retention was censored at the date of the last reported follow-up visit.
CDAI was considered both as a continuous outcome over time and as a measure of remission or low disease activity (LDA) at 1 year, using the validated thresholds. ${ }^{28} 29$ The frequency of assessments in most available registries did not allow for shorter evaluations of remission or LDA. We used the CDAI as a measure of disease activity instead of the DAS28 to avoid an assessment bias in favour of TCZ that has a strong impact of acute phase reactants. ${ }^{30}$ We also used the DAS28-eythrocyte sedimentation rate (ESR) as a secondary outcome measure.

\section{Covariates}

The baseline covariates considered were sex, age, disease duration, number of previously used bDMARDs, seropositivity (presence of rheumatoid factor (RF) or anticyclic citrullinated peptide antibodies), glucocorticoid (GC) use and daily dosage, functional disability (Health Assessment Questionnaire (HAQ)), DAS28-ESR, year of treatment initiation and country of registry. Seropositivity was operationally defined as positive if RF and/ or anticitrullinated protein antibody were positive according to each national registry, negative if both were negative and missing if one was missing and the other was negative. This algorithm is designed to limit misclassification of exposure and assign seronegative status to patients with missing data.

\section{Statistical methods}

Baseline characteristics across treatment were compared using generalised estimating equations, to account for the nested structure of the data, since patients could have several treatment courses and come from separate centres (registers). Drug retention was analysed using Kaplan-Meier and Cox models. In the Cox models, the baseline hazards were allowed to vary by country of registers and year of treatment initiation, and a cluster term was added to account for the fact that the same patients could have both TNFi and TCZ treatment. Missing covariates were imputed using multiple imputations with chained equations. CDAI and DAS28 change over time were analysed with mixed-effects models for longitudinal data. The frequency of disease remission or LDA under treatment was assessed at 1-year post-treatment start. When no observed values within a 3 -month window were available, they were interpolated using a quadratic interpolation for each patient. The proportions of patients reaching remission or LDA by treatment group were then estimated using frequency and proportion (raw estimates) and corrected for drug discontinuation using the LUNDEX index (index combining the proportion of patients fulfilling specific response criteria with the proportion of patients still adhering to therapy). ${ }^{31}$

\section{RESULTS}

A total of 8308 eligible treatment courses were retrieved before January 2017, including 771 TCZ mono, 1773 TCZ in combination therapy (TCZ combo) (87.5\% of all TCZ by intravenous administration), 1404 TNFi mono and 4660 TNFi combo. All registries contributed patients to both the TCZ and TNFi groups (mean proportion of TCZ patients across registries: 38.8\%, range: 9.2\%-73.7\%). Among TNFi patients, 24.8\% were on adalimumab, $15.1 \%$ on certolizumab, $34.2 \%$ on etanercept, $15.8 \%$ on golimumab and $10.0 \%$ on infliximab. On average, TCZ patients were slightly older, had longer disease duration and more previous bDMARDs. Baseline disease characteristics were slightly more severe in TCZ patients, with higher HAQ values, higher patient global assessment and higher $\mathrm{C}$ reactive protein levels (table 1). Patients in monotherapy (TCZ and 
Table 1 Patient characteristics at baseline

\begin{tabular}{|c|c|c|c|c|}
\hline & TCZ mono & TCZ combo & TNFi mono & TNFi combo \\
\hline $\mathrm{N}$ & 771 & 1773 & 1404 & 4660 \\
\hline Age, year (median, IQR) & $55.8(47.5,64.5)$ & $55.4(46.8,62.2)$ & $54.5(45.3,63.8)$ & $54.3(44.0,61.9)$ \\
\hline Female gender, N (\%) & $639(82.9 \%)$ & $1421(80.2 \%)$ & $1165(83.2 \%)$ & $3718(79.9 \%)$ \\
\hline Disease duration, year (median, IQR) & $10.2(4.6,17.2)$ & $9.0(4.3,15.3)$ & $8.7(3.7,15.6)$ & $7.9(3.4,14.6)$ \\
\hline Seropositivity (RF and/or ACPA), N (\%) & $458(83.7 \%)$ & $1134(83.0 \%)$ & $689(79.5 \%)$ & $2772(80.8 \%)$ \\
\hline \multicolumn{5}{|l|}{ Previous bDMARDs, N (\%) } \\
\hline 1 & $301(39.0 \%)$ & $737(41.6 \%)$ & $822(58.5 \%)$ & $3204(68.8 \%)$ \\
\hline 2 & $256(33.2 \%)$ & $572(32.3 \%)$ & $276(19.7 \%)$ & $641(13.8 \%)$ \\
\hline$\geq 3$ & $214(27.8 \%)$ & $464(26.2 \%)$ & $306(21.8 \%)$ & $815(17.5 \%)$ \\
\hline Glucocorticoids, N (\%) & $193(25.0 \%)$ & $893(50.4 \%)$ & $308(21.9 \%)$ & $2625(56.3 \%)$ \\
\hline Glucocorticoids dose, mg/day (median, IQR) & $5.0(4.0,10.0)$ & $5.0(5.0,10.0)$ & $5.0(5.0,7.5)$ & $5.0(5.0,7.5)$ \\
\hline \multicolumn{5}{|l|}{ Concomitant csDMARD, N (\%) } \\
\hline MTX & - & $931(52.5 \%)$ & - & $2097(45.0 \%)$ \\
\hline MTX+other & & $363(20.5 \%)$ & & $1421(30.5 \%)$ \\
\hline Other & & $479(27.0 \%)$ & & $1142(24.5 \%)$ \\
\hline DAS28 & $4.1(1.7)$ & $4.6(1.4)$ & $4.1(1.4)$ & $4.0(1.4)$ \\
\hline CDAI & $23.3(15.9)$ & $27.7(14.8)$ & $22.6(15.3)$ & $21.4(14.4)$ \\
\hline HAQ & $1.3(0.7)$ & $1.4(0.7)$ & $1.2(0.8)$ & $1.1(0.8)$ \\
\hline TJC (over 28 joints) & $7.7(7.2)$ & $9.1(6.8)$ & $7.5(6.9)$ & $6.8(6.3)$ \\
\hline SJC (over 28 joints) & $6.0(6.0)$ & $7.4(6.0)$ & $5.9(5.9)$ & $5.9(5.6)$ \\
\hline ESR (mm/hour) & $29.6(25.5)$ & $34.4(26.7)$ & $27.6(23.8)$ & $26.5(22.6)$ \\
\hline CRP (mg/L) & $16.7(26.8)$ & $19.9(26.6)$ & $16.4(25.5)$ & $14.9(21.4)$ \\
\hline Patient global assessment & $5.5(2.8)$ & $6.0(2.5)$ & $5.5(2.7)$ & $5.1(2.7)$ \\
\hline Physician global assessment & $4.1(2.5)$ & $5.1(2.4)$ & $4.2(2.5)$ & $4.0(2.4)$ \\
\hline
\end{tabular}

Values are mean (SD) when not specified.

ACPA, anticitrullinated protein antibody; bDMARD, biologic disease-modifying antirheumatic drugs; CDAl, Clinical Disease Activity Index; combo, combination therapy; CRP, C reactive protein; CSDMARD, conventional synthetic DMARD; DAS28, Disease Activity Score 28; ESR, erythrocyte sedimentation rate; HAQ, Health Assessment Questionnaire; mono, monotherapy; MTX, methotrexate; RF, rheumatoid factor; SJC, swollen joint count; TCZ, tocilizumab; TJC, tender joint count; TNFi, tumour necrosis factor inhibitor.

TNFi) had less GCs than patients in combination therapy. Baseline characteristics of patients of the subanalysis by dose of MTX are in online supplementary table S2.

Crude median drug retention (figure 1) was higher for TCZ mono (2.31 years, 95\% CI 2.07 to 2.61 ) or combo (1.98 years,

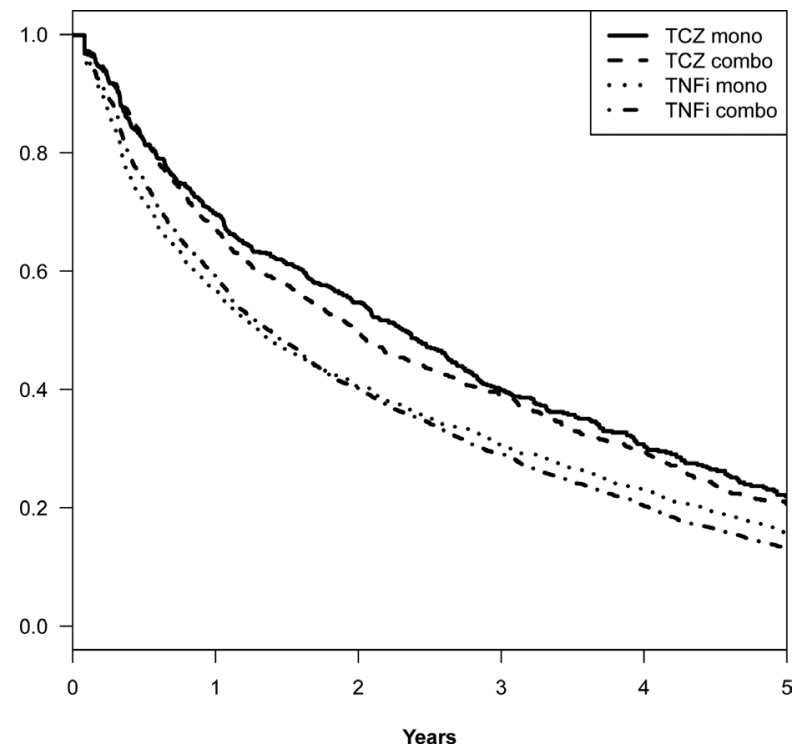

Figure 1 Kaplan-Meier curves of drug discontinuation by biologics and presence or not of concomitant conventional synthetic diseasemodifying antirheumatic drugs (csDMARDs). TCZ combo, tociluzimab in combination with csDMARDs; TCZ mono, tocilizumab as monotherapy; TNFi combo, TNF inhibitor in combination with csDMARDs; TNFi mono, TNF inhibitor as monotherapy.
95\% CI 1.83 to 2.11$)$ than TNFi combo $(1.37$ years, $95 \% \mathrm{CI}$ 1.30 to 1.45 ) or mono (1.31 years, $95 \%$ CI 1.18 to 1.47$)$. Among TNFi combo patients, crude median retention by concomitant csDMARD was 1.55 years (95\% CI 1.43 to 1.64$)$ for MTX, 1.36 years (95\% CI 1.24 to 1.57$)$ for MTX +another csDMARD and 1.09 years (95\% CI 1.00 to 1.23 ) for csDMARD other than MTX (see online supplementary figure S1A). Among TNFi combo patients with MTX as the only concomitant csDMARD (n with available MTX dose=1520), median drug retention was 1.27 years (95\% CI 1.04 to 1.62) for patients with low dose of MTX $(<10 \mathrm{mg} /$ week, $\mathrm{n}=170), 1.33$ years $(95 \% \mathrm{CI} 1.10$ to 1.57$)$ for patients with medium dose of MTX $(10-15 \mathrm{mg} / \mathrm{week}, \mathrm{n}=697)$ and 1.64 years (95\% CI 1.44 to 1.82 ) for patients with high dose ( $>15 \mathrm{mg} /$ week, $\mathrm{n}=653$ ) (see online supplementary figure S1B).

In a country and year of treatment initiation-stratified, covariate-adjusted analysis, we found that hazards of discontinuation of TCZ mono or combo were significantly lower than for TNFi mono or combo, and lower for TNFi combo than mono but similar between TCZ mono and combo (table 2).

When comparing TCZ mono with TNFi combo, TCZ mono was stopped more frequently for ineffectiveness than TNFi combo (24.0\% vs $13.9 \%)$, whereas discontinuation was equally often recorded for adverse events in TCZ mono-treated and TNFi combo-treated patients (13.0\% vs $13.6 \%)$. However, most of the causes of treatment discontinuation were recorded as 'other' by the treating physicians, which may include patient preference, remission and pregnancy, as well as a combination of causes $(\mathrm{TCZ}$ mono $=56.0 \%$, TNFi combo $=42.6 \%)$. Among the TCZ mono group, 50 treatment courses $(6.5 \%)$ were changed to include a concomitant csDMARD at some point. Conversely, among the TNFi combo group, 313 treatment courses $(6.7 \%)$ 
Table 2 Multivariable analysis of drug discontinuation.

\begin{tabular}{llll}
\hline & HR & $95 \%$ Cl & P values \\
\hline TCZ mono vs TNFi combo & 0.78 & 0.70 to 0.86 & $<0.001$ \\
\hline TNFi mono vs TNFi combo & 1.15 & 1.06 to 1.23 & $<0.001$ \\
TCZ mono vs TCZ combo & 0.96 & 0.86 to 1.08 & 0.53 \\
\hline TCZ mono vs TNFi mono & 0.65 & 0.58 to 0.74 & $<0.001$ \\
\hline TCZ combo vs TNFi combo & 0.70 & 0.65 to 0.76 & $<0.001$ \\
\hline TCZ combo vs TNFi mono & 0.65 & 0.59 to 0.72 & $<0.001$ \\
\hline
\end{tabular}

Adjusted by age, gender, disease duration, seropositivity, number of previous biologic disease-modifying antirheumatic drugs, glucocorticoids at baseline, Disease Activity Score 28 at baseline, Clinical Disease Activity Index at baseline, Health Assessment Questionnaire at baseline.

combo, combination therapy; mono, monotherapy; TCZ, tocilizumab; TNFi, tumour necrosis factor inhibitor.

were modified to stop the csDMARD for at least some visits (more than one-fourth of the visits). Shorter disease duration, higher past number of bDMARDs, concomitant GC treatment and higher HAQ at baseline were significantly associated with greater risk of discontinuation (see online supplementary table S3). The hazards of discontinuation were also significantly lower when comparing TCZ mono patients (HR 0.75, p<0.001) with TNFi patients treated with a high dose of MTX (>15 mg/week) (see online supplementary table S3, right columns).

CDAI score significantly decreased over time in the four different groups, and the decrease was not significantly different between them (table 3 ). The average adjusted CDAI change at 1 year was of -3.54 for TNFi mono patients, -3.34 for TNFi combo patients, -3.68 for TCZ combo and -3.58 for TCZ mono patients. When comparing TCZ mono versus TNFi combo, shorter disease duration, higher number of past bDMARDs, higher HAQ at baseline and concomitant GC treatment were associated with higher CDAI at any time during follow-up (see online supplementary table S4). The pattern of findings was similar when comparing TCZ mono patients with TNFi patients treated with a high dose of MTX (see online supplementary table S4, right columns), though number of past bDMARDs became non-significant.

Two hundred and fifty-one TCZ mono, 737 TCZ combo, 375 TNFi mono and 1995 TNFi combo patients were still under treatment with CDAI information at 1 year and could be included in the LUNDEX calculation. CDAI rates were relatively similar between groups, although CDAI LDA rates seemed lower in TNFi mono patients. However, this trend was not reflected in the CDAI remission rates (figure 2). In contrast, DAS28 remission and LDA at 1 year (LUNDEX corrected) were considerably higher in TCZ patients compared with TNFi patients (see online supplementary figure S2). For TCZ mono vs TNFi in combination

Table 3 Multivariable analysis of CDAI over time

\begin{tabular}{lrll}
\hline & Coeff & 95\% Cl & P values \\
\hline TCZ mono vs TNFi combo & 0.17 & -1.33 to 1.66 & 0.83 \\
\hline TNFi mono vs TNFi combo & -0.23 & -1.06 to 0.60 & 0.59 \\
TCZ mono vs TCZ combo & -0.21 & -1.24 to 0.83 & 0.70 \\
\hline TCZ mono vs TNFi mono & -0.47 & -1.60 to 0.66 & 0.41 \\
\hline TCZ combo vs TNFi combo & 0.09 & -0.56 to 0.74 & 0.79 \\
\hline TCZ combo vs TNFi mono & 0.21 & -0.74 to 1.16 & 0.67 \\
\hline
\end{tabular}

Adjusted by age, gender, disease duration, seropositivity, number of previous biologic disease-modifying antirheumatic drugs, glucocorticoids at baseline, Health Assessment Questionnaire at baseline.

Coeff, coefficient; combo, combination therapy; mono, monotherapy; TCZ, tocilizumab; TNFi, tumour necrosis factor inhibitor. with MTX, across doses of MTX, CDAI remission and LDA rates remained similar after LUNDEX adjustment (see online supplementary figure S2). They also remained similar across the type of concomitant csDMARD with TNFi. Conversely, DAS28 remission and LDA rates were higher in TCZ mono than for TNFi patients, across type of concomitant csDMARD or MTX doses after LUNDEX adjustment (see online supplementary figure S4).

\section{DISCUSSION}

Our study is one of the largest comparing TCZ and TCZ mono and TCZ combo, and one of the first that compared TCZ mono versus TNFi combo in a large population of patients with RA who used at least one bDMARD. The results showed that drug retention was significantly longer with TCZ than TNFi, even when TCZ was used as monotherapy and TNFi in combination therapy. The clinical effectiveness, as assessed by CDAI changes and CDAI responses, were similar in all treatment groups. In contrast, as expected, changes in DAS2 8 and DAS2 8 responses were significantly better in TCZ than in TNFi-treated patients. Altogether, the results indicate that TCZ mono or combination therapy are valuable therapeutic option in patients with an inadequate response to bDMARDs.

The patient populations differed in terms of baseline characteristics with older patients, longer disease duration, higher HAQ and more previous bDMARD failure in the TCZ groups. These results are consistent with other studies showing that bDMARDs as monotherapy are usually prescribed to more difficult-to-treat patients. ${ }^{9} 1032$ However, despite these differences, drug effectiveness as assessed by CDAI was similar in the two groups, whereas drug retention was longer with TCZ compared with TNFi whatever the mode of administration (monotherapy or combination therapy). Patients in monotherapy either with TCZ or TNFi had also less GCs than patients in combination therapy, indicating that patients treated as monotherapy have a different profile in terms of disease characteristics and comorbidities. However, the results regarding drug retention and efficacy were adjusted for the use of GCs.

Although bDMARD retention was higher in TCZ than in TNFi-treated patients, the effectiveness of these treatments, based on the CDAI, was not significantly different. This discrepancy suggests that either CDAI does not allow a comprehensive assessment of drug efficacy, the presence of a difference of tolerance between the two treatment groups, which was apparently not identified in our study, or that retention probably captures something that is not evaluated by CDAI, such as patient or physician preference. For example, it is possible that some minor adverse events, not recorded in the registries but sufficient to discourage patients to continue their treatment, can partly account for the difference. It is also possible that TCZ treatment, being used after several bDMARDs failure, is maintained even if not achieving the ideal target due to the lack of treatment alternatives.

Considering similar effectiveness in terms of CDAI and retention to the TCZ combination therapy, TCZ mono may also be suitable for patients who cannot tolerate csDMARDs or in whom these treatments are contraindicated. Indeed, although MTX is still the mainstay of RA therapy, up to $30 \%$ of patients discontinue MTX because of preference ${ }^{33-35}$ or toxic effects. ${ }^{3637}$ Thus, there is a need to provide patients with effective alternatives. As consistently reported, contrary to monotherapy with either adalimumab or etanercept, the efficacy of TCZ mono is higher than MTX alone. ${ }^{623}$ Furthermore, TCZ mono was superior to adalimumab as monotherapy in patients with inadequate 

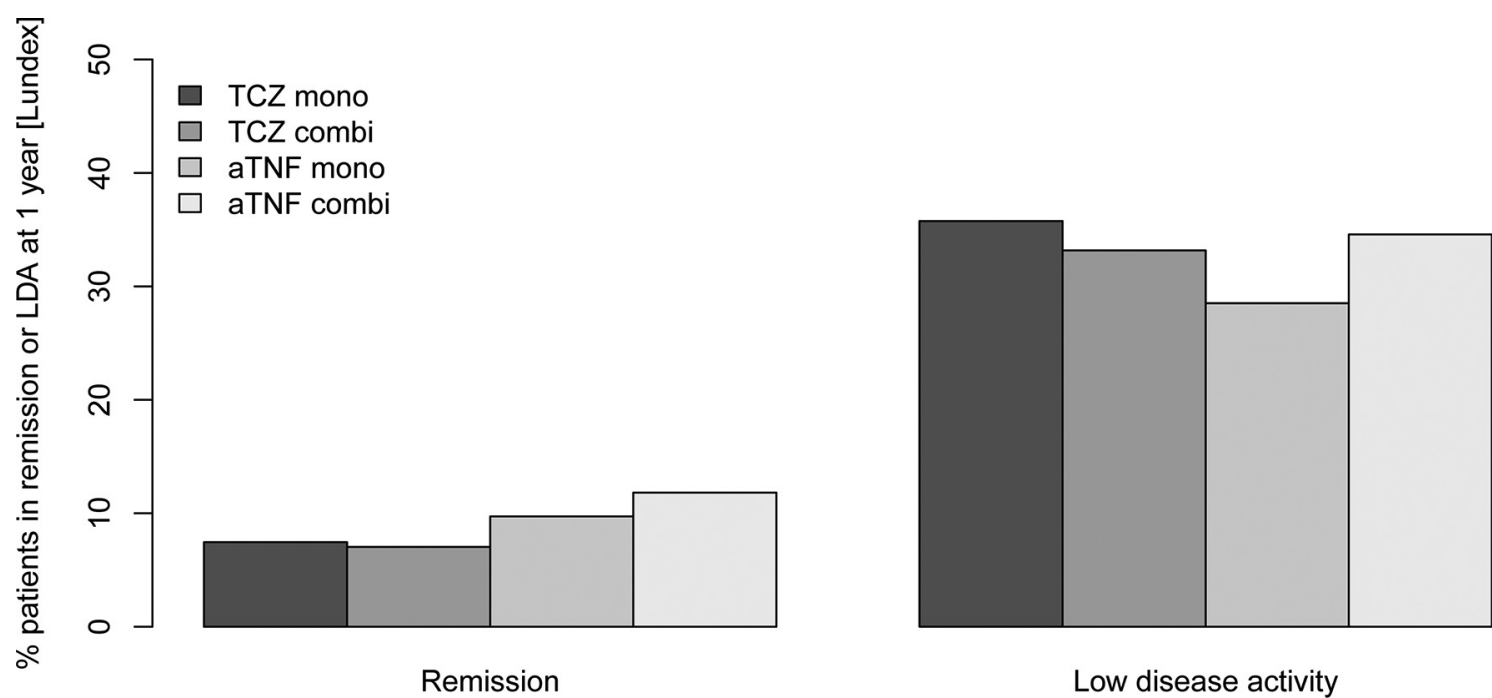

\section{Remission}

Low disease activity

Figure 2 Clinical Disease Activity Index (CDAl) remission or low disease activity (LDA) at 1 year by biologics and presence or not of concomitant conventional synthetic disease-modifying antirheumatic drugs (csDMARDs). Two hundred and fifty-one TCZ mono, 737 TCZ combo, 375 TNFi mono and 1995 TNFi combo patients were still under treatment with CDAI information at 1 year and could be included in the LUNDEX calculation. TCZ combo, tocilizumab in combination with csDMARDs; TCZ mono, tocilizumab as monotherapy; TNFi combo, TNF inhibitors in combination with csDMARDs; TNFi mono, TNF inhibitors as monotherapy.

response to MTX or in whom MTX was not appropriate. Recent data from another head-to-head trial comparing adalimumab with sarilumab, another anti-IL-6R antibody, showed that in monotherapy sarilumab is superior to adalimumab. ${ }^{38}$ These results indicate that IL- 6 receptor antagonists have an advantage over TNFi when prescribed as monotherapy. Consistent with these findings, the current European League Against Rheumatism recommendations for the management of RA, mention that IL-6 inhibitors and Janus kinase inhibitors may have some advantage over other bDMARDs, if patients cannot use csDMARD. ${ }^{8}$ The ACT-iON study has shown that, after an inadequate response to csDMARDs, the efficacy and retention of intravenous TCZ was better than TNFi, both given mostly in combination therapy. ${ }^{39}$ However, none of these studies compared TCZ mono with TNFi combo. Furthermore, these studies did not include patients previously exposed to TNFi and other bDMARDs.

The rate of TNFi retention was higher in patients receiving TNFi in combination with MTX alone than with other csDMARD. Similarly, patients treated with the highest MTX doses had longer treatment maintenance than those receiving TNFi with lower MTX doses. The superiority of MTX versus other csDMARDs is consistent with the results of previous clinical trials and observational studies. ${ }^{1040} 41$ Furthermore, MTX has been shown to have a dose-dependent positive influence on adalimumab efficacy. ${ }^{42}$ However, in the covariate-adjusted analysis, hazards of discontinuation were still lower with TCZ mono than in TNFi in combination with the highest dose of MTX (>15 mg weekly) (table 2).

We found no difference in the change of CDAI over time between TCZ and TNFi, whereas effects on DAS28 were significantly different between groups, with more patients treated with TCZ achieving remission or LDA. This finding is consistent with the effect of IL- 6 blockade on acute-phase reactants. ${ }^{30}$

Our study has several limitations. We took into account only patients who previously used at least one bDMARD, which may reduce the external validity of our findings. However, our results are relevant to clinical practice since TCZ, especially as monotherapy, is commonly prescribed in patients who had previously been exposed to bDMARDs. Because of the observational nature of our data, we cannot exclude potential unmeasured confounders in the baseline characteristics for which we cannot adjust. In particular, only a few registries captured comorbidities and we could not include them in our analysis. The recording of causes of discontinuation in the registries were also not detailed enough to allow further analysis, with the great majority accounted as 'other reason' than lack of effectiveness or adverse events. Another consequence of the observational nature of the data is that patients, initially classified as combo or mono according to baseline data, may have either stopped the csDMARD or started a csDMARD over time. However, this misclassification bias of the exposure seemed relatively small ( $\sim 7 \%$ for TCZ mono and TNFi combo). Finally, several studies reported poor adherence to MTX and under-recognition of this phenomenon by treating physicians. ${ }^{43} 44$ Unfortunately, our data do not allow us to evaluate the importance of this phenomenon. The strengths of our study are that we have a large sample of patients with a long duration of follow-up and detailed data on clinical end points. The observational setting also allows the inclusion of a diverse population of patients from different countries without the strict inclusion criteria generally used in randomised controlled trial.

In conclusion, our results support that TCZ mono or in combination with csDMARDs are reasonable therapeutic options in patients with inadequate response to at least one bDMARD, with similar effectiveness in terms of CDAI to the TNFi combination therapy but longer retention.

\footnotetext{
Author affiliations

${ }^{1}$ Geneva University Hospitals, Geneva, Switzerland

${ }^{2} \mathrm{SCQM}$ Registry, Zurich, Switzerland

${ }^{3}$ ROB-FIN Helsinki University Hospital and Helsinki University, Helsinki, Finland

${ }^{4}$ Charles University, Prague, Czech Republic

${ }^{5}$ Rheumatology Department, Hospital Clinic Barcelona, Barcelona, Spain

${ }^{6}$ Department of Rheumatology, Diakonhjemmet Hospital, Oslo, Norway

${ }^{7}$ Rheumatology Department, Hospital Garcia de Orta, on behalf of Reuma.pt, Almada, Portugal

${ }^{8}$ BioRx.si, University Medical Centre Ljubljana, Ljubljana, Slovenia

${ }^{9} \mathrm{GISEA}$, University Hospital of Bari, Bari, Italy

${ }^{10}$ Center of Rheumatic Diseases, University of Medicine and Pharmacy, Bucharest, Romania

${ }^{11}$ ARBITER, Institute of Rheumatology, Moscow, Russian Federation
} 
${ }^{12}$ Genentech, South San Francisco, California, USA

${ }^{13} \mathrm{~F}$. Hoffmann-La Roche AG, Basel, Switzerland

Acknowledgements The authors thank all the rheumatologists and patients who participated in the registries.

Contributors All the authors have provided substantial contributions to the conception or design of the work, the acquisition of the data and the interpretation of data. DSC and KL performed the statistical analysis. KL, DSC and CG made the first draft. All the other authors participated in the final drafting of the work or revising it critically for important intellectual content. All authors contributed to the final approval of the version published.

Funding The TOCERRA collaboration is funded by Roche.

Competing interests Swiss Clinical Quality Management in Rheumatic Diseases (SCQM) database is sponsored by public and industrial support (http://scam.ch/en/ sponsoren/). DCN benefited from grant and research support from AbbVie, BMS, MSD, Pfizer, Roche and UCB, and has received fees for speaking and/or consulting for AbbVie, BMS, MSD, Roche, UCB and Pfizer. ROB-FIN is funded by AbbVie, Hospira, BMS, MSD, Pfizer, Roche and UCB. KP benefited from grant and research support from AbbVie, Roche, Medis, MSD and Pfizer and has received fees for speaking and/or consulting for AbbVie, Roche, Amgen, MSD, BMS, UCB and Egis. Clinical work in Czech Republic was supported by the project from the Ministry of Health for conceptual development of research organisation 023728 (Institute of Rheumatology). TKK has received fees for speaking and/or consulting from AbbVie, BMS, Celgene, Celltrion, Eli Lilly, Hospira, Merck-Serono, MSD, Orion Pharma, Pfizer, Roche, Sandoz and UCB and received research funding to Diakonhjemmet Hospital from AbbVie, BMS, MSD, Pfizer, Roche and UCB. NOR-DMARD was previously supported with research funding to Diakonhjemmet Hospital from AbbVie, BMS, MSD/Schering-Plough, Pfizer/Wyeth, Roche and UCB. Reuma.pt is supported by unrestricted grants from AbbVie, MSD, Roche and Pfizer. ŽR: none declared. BioRx.si has received funding for clinical research paid to Društvo za razvoj revmatologije from AbbVie, Roche, Medis, MSD and Pfizer. CC: has received speaker and consulting fees from AbbVie, Amgen, Angellini, AstraZeneca, BMS, Egis, MSD, Pfizer, Richter, Roche, Sanofi, Servier, Teva, UCB, Zentiva. GL has received fees for consulting for BMS, Roche, MSD, AbbVie and Pfizer. The ARBITER registry is supported by a non-commercial partnership with 'Equalrights to life'. SLG and KS are employees of Genentech. YL is an employee of F. Hoffmann-La Roche. DSC has received consulting fees from BMS, Pfizer and Janssen. CG has received fees for speaking and/or consulting from AbbVie, BMS, Roche, Pfizer, Celgene, MSD, Janssen Cilag, Amgen, UCB and received research funding from Roche, AbbVie, MSD and Pfizer.

\section{Patient consent Not required.}

Ethics approval Approval of each local ethical committee for the collection of clinical data in each registry.

Provenance and peer review Not commissioned; externally peer reviewed.

Data sharing statement All the data belong to the registries. Anonymised data can be shared as long as agreements are made with all participating registries.

(c) Article author(s) (or their employer(s) unless otherwise stated in the text of the article) 2018. All rights reserved. No commercial use is permitted unless otherwise expressly granted.

\section{REFERENCES}

1 Nishimoto N, Hashimoto J, Miyasaka N, et al. Study of active controlled monotherapy used for rheumatoid arthritis, an IL-6 inhibitor (SAMURAI): evidence of clinical and radiographic benefit from an $\mathrm{x}$ ray reader-blinded randomised controlled trial of tocilizumab. Ann Rheum Dis 2007;66:1162-7.

2 Maini RN, Taylor PC, Szechinski J, et al. Double-blind randomized controlled clinical trial of the interleukin-6 receptor antagonist, tocilizumab, in European patients with rheumatoid arthritis who had an incomplete response to methotrexate. Arthritis Rheum 2006;54:2817-29.

3 Kremer JM, Blanco R, Brzosko M, et al. Tocilizumab inhibits structural joint damage in rheumatoid arthritis patients with inadequate responses to methotrexate: results from the double-blind treatment phase of a randomized placebo-controlled trial of tocilizumab safety and prevention of structural joint damage at one year. Arthritis Rheum 2011;63:609-21.

4 Emery P, Keystone E, Tony HP, et al. IL-6 receptor inhibition with tocilizumab improves treatment outcomes in patients with rheumatoid arthritis refractory to anti-tumour necrosis factor biologicals: results from a 24-week multicentre randomised placebocontrolled trial. Ann Rheum Dis 2008:67:1516-23.

5 Smolen IS, Beaulieu A, Rubbert-Roth A, et al. Effect of interleukin-6 receptor inhibition with tocilizumab in patients with rheumatoid arthritis (OPTION study): a double-blind, placebo-controlled, randomised trial. Lancet 2008;371:987-97.

6 Jones G, Sebba A, Gu J, et al. Comparison of tocilizumab monotherapy versus methotrexate monotherapy in patients with moderate to severe rheumatoid arthritis: the AMBITION study. Ann Rheum Dis 2010;69:88-96.
7 Summary of product characteristics RoActemra. http://www.ema.europa.eu/docs/en GB/document_library/EPAR_-_Product_Information/human/000955/WC500054890. pdf.

8 Smolen JS, Landewé R, Biilsma J, et al. EULAR recommendations for the management of rheumatoid arthritis with synthetic and biological disease-modifying antirheumatic drugs: 2016 update. Ann Rheum Dis 2017;76:960-77.

9 Gabay C, Riek M, Hetland ML, et al. Effectiveness of tocilizumab with and without synthetic disease-modifying antirheumatic drugs in rheumatoid arthritis: results from a European collaborative study. Ann Rheum Dis 2016;75:1336-42.

10 Soliman MM, Ashcroft DM, Watson KD, et al. Impact of concomitant use of DMARDs on the persistence with anti-TNF therapies in patients with rheumatoid arthritis: results from the British Society for Rheumatology Biologics Register. Ann Rheum Dis 2011;70:583-9.

11 Mariette X, Gottenberg JE, Ravaud P, et al. Registries in rheumatoid arthritis and autoimmune diseases: data from the French registries. Rheumatology 2011;50:222-9.

12 Lee SJ, Chang H, Yazici Y, et al. Utilization trends of tumor necrosis factor inhibitors among patients with rheumatoid arthritis in a United States observational cohort study. I Rheumatol 2009;36:1611-7.

13 Heiberg MS, Koldingsnes W, Mikkelsen K, et al. The comparative one-year performance of anti-tumor necrosis factor alpha drugs in patients with rheumatoid arthritis, psoriatic arthritis, and ankylosing spondylitis: results from a longitudinal, observational, multicenter study. Arthritis Rheum 2008;59:234-40.

14 Yazici Y, Shi N, John A. Utilization of biologic agents in rheumatoid arthritis in the United States: analysis of prescribing patterns in 16,752 newly diagnosed patients and patients new to biologic therapy. Bull NYU Hosp Jt Dis 2008;66:77-85.

15 Askling J, Fored CM, Brandt L, et al. Time-dependent increase in risk of hospitalisation with infection among Swedish RA patients treated with TNF antagonists. Ann Rheum Dis 2007;66:1339-44.

16 Listing J, Strangfeld A, Rau R, et al. Clinical and functional remission: even though biologics are superior to conventional DMARDs overall success rates remain lowresults from RABBIT, the German biologics register. Arthritis Res Ther 2006;8:R66.

17 Dougados M, Kissel K, Sheeran T, et al. Adding tocilizumab or switching to tocilizumab monotherapy in methotrexate inadequate responders: 24-week symptomatic and structural results of a 2-year randomised controlled strategy trial in rheumatoid arthritis (ACT-RAY). Ann Rheum Dis 2013;72:43-50.

18 Dougados M, Kissel K, Conaghan PG, et al. Clinical, radiographic and immunogenic effects after 1 year of tocilizumab-based treatment strategies in rheumatoid arthritis: the ACT-RAY study. Ann Rheum Dis 2014;73:803-9.

19 Huizinga TW, Conaghan PG, Martin-Mola E, et al. Clinical and radiographic outcomes at 2 years and the effect of tocilizumab discontinuation following sustained remission in the second and third year of the ACT-RAY study. Ann Rheum Dis 2015;74:35-43.

20 Kaneko Y, Atsumi T, Tanaka Y, et al. Comparison of adding tocilizumab to methotrexate with switching to tocilizumab in patients with rheumatoid arthritis with inadequate response to methotrexate: 52 -week results from a prospective, randomised, controlled study (SURPRISE study). Ann Rheum Dis 2016;75:1917-23.

21 Burmester GR, Rigby WF, van Vollenhoven RF, et al. Tocilizumab in early progressive rheumatoid arthritis: FUNCTION, a randomised controlled trial. Ann Rheum Dis 2016;75:1081-91.

22 Gabay C, Emery P, van Vollenhoven R, et al. Tocilizumab monotherapy versus adalimumab monotherapy for treatment of rheumatoid arthritis (ADACTA): a randomised, double-blind, controlled phase 4 trial. Lancet 2013;381:1541-50.

23 Klareskog L, van der Heijde D, de Jager JP, et al. Therapeutic effect of the combination of etanercept and methotrexate compared with each treatment alone in patients with rheumatoid arthritis: double-blind randomised controlled trial. Lancet 2004;363:675-81.

24 Goekoop-Ruiterman YP, de Vries-Bouwstra JK, Allaart CF, et al. Clinical and radiographic outcomes of four different treatment strategies in patients with early rheumatoid arthritis (the BeSt study): a randomized, controlled trial. Arthritis Rheum 2005:52:3381-90.

25 Breedveld FC, Weisman MH, Kavanaugh AF, et al. The PREMIER study: A multicenter, randomized, double-blind clinical trial of combination therapy with adalimumab plus methotrexate versus methotrexate alone or adalimumab alone in patients with early, aggressive rheumatoid arthritis who had not had previous methotrexate treatment. Arthritis Rheum 2006:54:26-37.

26 Pincus T, Marcum SB, Callahan LF. Longterm drug therapy for rheumatoid arthritis in seven rheumatology private practices: II. Second line drugs and prednisone. J Rheumatol 1992;19:1885-94.

27 Fisher A, Bassett K, Wright JM, et al. Comparative persistence of the TNF antagonists in rheumatoid arthritis--a population-based cohort study. PLoS One 2014;9:e105193.

28 Aletaha D, Smolen J. The Simplified Disease Activity Index (SDAI) and the Clinical Disease Activity Index (CDAI): a review of their usefulness and validity in rheumatoid arthritis. Clin Exp Rheumatol 2005;23:S100-8.

29 Aletaha D, Nell VP, Stamm T, et al. Acute phase reactants add little to composite disease activity indices for rheumatoid arthritis: validation of a clinical activity score. Arthritis Res Ther 2005:7:R796-806.

30 Smolen JS, Aletaha D. Interleukin-6 receptor inhibition with tocilizumab and attainment of disease remission in rheumatoid arthritis: the role of acute-phase reactants. Arthritis Rheum 2011;63:43-52. 
31 Kristensen LE, Saxne T, Geborek P. The LUNDEX, a new index of drug efficacy in clinical practice: results of a five-year observational study of treatment with infliximab and etanercept among rheumatoid arthritis patients in southern Sweden. Arthritis Rheum 2006:54:600-6.

32 Gabay C, Riek M, Scherer A, et al. Effectiveness of biologic DMARDs in monotherapy versus in combination with synthetic DMARDs in rheumatoid arthritis: data from the Swiss Clinical Quality Management Registry. Rheumatology 2015;54:1664-72.

33 Grijalva CG, Kaltenbach L, Arbogast PG, et al. Adherence to disease-modifying antirheumatic drugs and the effects of exposure misclassification on the risk of hospital admission. Arthritis Care Res 2010;62:730-4.

34 de Thurah A, Nørgaard M, Harder I, et al. Compliance with methotrexate treatment in patients with rheumatoid arthritis: influence of patients' beliefs about the medicine. A prospective cohort study. Rheumatol Int 2010;30:1441-8.

35 Cannon GW, Mikuls TR, Hayden CL, et al. Merging Veterans Affairs rheumatoid arthritis registry and pharmacy data to assess methotrexate adherence and disease activity in clinical practice. Arthritis Care Res 2011;63:1680-90.

36 Nikiphorou E, Negoescu A, Fitzpatrick JD, et al. Indispensable or intolerable? Methotrexate in patients with rheumatoid and psoriatic arthritis: a retrospective review of discontinuation rates from a large UK cohort. Clin Rheumatol 2014;33:609-14.

37 Salliot C, van der Heijde D. Long-term safety of methotrexate monotherapy in patients with rheumatoid arthritis: a systematic literature research. Ann Rheum Dis 2009:68:1100-4.
38 Burmester GR, Lin Y, Patel R, et al. Efficacy and safety of sarilumab monotherapy versus adalimumab monotherapy for the treatment of patients with active rheumatoid arthritis (MONARCH): a randomised, double-blind, parallel-group phase III trial. Ann Rheum Dis 2017;76:840-7.

39 Choy EH, Bernasconi C, Aassi M, et al. Treatment of Rheumatoid Arthritis With Anti-Tumor Necrosis Factor or Tocilizumab Therapy as First Biologic Agent in a Global Comparative Observational Study. Arthritis Care Res 2017;69:1484-94.

40 Burmester GR, Mariette X, Montecucco C, et al. Adalimumab alone and in combination with disease-modifying antirheumatic drugs for the treatment of rheumatoid arthritis in clinical practice: the Research in Active Rheumatoid Arthritis (ReAct) trial. Ann Rheum Dis 2007;66:732-9.

41 Aaltonen KJ, Joensuu JT, Pirilä L, et al. Drug survival on tumour necrosis factor inhibitors in patients with rheumatoid arthritis in Finland. Scand I Rheumatol 2017;46:1-5.

42 Burmester GR, Kivitz AJ, Kupper H, et al. Efficacy and safety of ascending methotrexate dose in combination with adalimumab: the randomised CONCERTO trial. Ann Rheum Dis 2015:74:1037-44.

43 Curtis JR, Bykerk VP, Aassi M, et al. Adherence and Persistence with Methotrexate in Rheumatoid Arthritis: A Systematic Review. J Rheumatol 2016:43:1997-2009.

44 Curtis JR, Bharat A, Chen L, et al. Agreement between Rheumatologist and Patientreported Adherence to Methotrexate in a US Rheumatoid Arthritis Registry. J Rheumatol 2016:43:1027-9. 